\title{
Increase of the Annual Energy Output in Hydraulic Powerplants through Active Flow Control
}

\author{
M.V. Magnoli ${ }^{1}$ and R. Schilling ${ }^{1}$ \\ ${ }^{1}$ Institute of Fluid Mechanics \\ Munich University of Technology \\ 85747 Garching, Germany \\ Phone/Fax number: +49 8928916297 \\ E-mail: magnoli@flm.mw.tum.de, schilling@flm.mw.tum.de
}

\begin{abstract}
Traditionally, hydraulic powerplants have been strongly contributing to energy generation. At present, with the expanding participation of other non-storable renewable energy sources in the electrical market composition, water turbines can importantly contribute to the electrical grid stability and to energy storage. With the increasing demand for such services, new procedures have to be developed, in order to allow the hydraulic turbines to operate in critical load conditions, as part load, for example. The technique proposed here, based on pitching guide vanes, was numerically tested with instationary CFD models and delivered possibly promising results, concerning the machine performance and stability, at part load conditions. The extension of the operating range of hydraulic powerplants, through the turbine active flow control, yields even greater flexibility to them in providing regulation services to the electrical grid. This technique has also the potential to extend the operational life of main turbine components and may lead to the increase of the annual energy output in some cases.
\end{abstract}

\section{Keywords}

Hydraulic turbines, instationary fluid flow, active flow control, turbulence modelling, numerical simulation.

\section{Introduction}

Waterpower corresponds to one of the earliest sources of renewable energy in commercial use. Still current days, it corresponds to an important part of the installed energy generation facilities, ranging from small complexes with less than $1 \mathrm{MW}$ up to Itaipu, in Brazil, and Three Gorges, in China, with rated capacity of, respectively, 14,0 GW and 18,2 GW and annual energy output of, respectively, 91,6 TWh and 79,4 TWh in 2009.
Most recently, with the increasing demand for clean energy sources, hydraulic energy appears once again as an interesting solution. In the current context of the energy market, where other renewable energy sources, such as wind and solar energy, enjoy increasing market shares, the hydraulic powerplants offer a very attractive solution for grid stabilization and energy storage. The energy output of wind and solar energy parks is influenced by variations of the weather conditions, sometimes producing more or less energy as in the moment needed and introducing a strong dynamic component in the electrical grid. With their increasing importance, their impact on the grid tends to be even greater, as well as the need for regulation and storage services. Hydraulic turbines can efficiently supply this dynamic balance to the electrical grid and in a faster way than traditional thermal plants. However, with the increasing need for such services, water turbines must also offer increasing flexibility.

At this point, it comes to the current challenge in the design of water turbines. The operating range of the hydraulic plants must be extended, in order to offer more possibilities in the regulation of energy generation as whole. It means, that the turbines must be able to be smoothly operated along a larger portion of their hill chart than in the past. Common customer requirements for modern water turbines are, for example, that they shall be capable to operate at deep part load, sometimes even considerably below the half of their rated power.

At deep part load in hydraulic turbines, with less than $60 \%$ of the optimal flow, $Q<0,60 Q_{\text {opt }}$, strong pressure pulsations, with amplitudes up to $10 \%$ of the nominal head, may arise in the machine, typically due to the formation of an oscillating vortex rope in the draft tube cone. The pressure pulsations are diffused through the hydraulic active parts of the turbine. In the case of strong pressure oscillations, they may affect 
the stability of the energy output at the generator, due to possibly strong torque oscillations, as well as they may cause structural damages to turbine components, especially to the turbine runner. This critical condition turns out to be even more challenging, if considered that the power density of water turbines has got much larger during the last decades, in order to satisfy economical and competitive needs.

There are different approaches to overcome the pressure oscillation problem. Common actions to reduce the amplitude of the pressure pulsations at part load, which are normally employed and which have been empirically established, are the air injection in the turbine or the installation of fins at the walls of the draft tube cone. Both solutions often impact negatively at the turbine efficiency and rely on weak theoretical backgrounds. Another solution would be to better understand the dynamical behaviour of the turbine at highly transient operating conditions, as done by Magnoli And Schilling [1], and adapt its mechanical design accordingly, including it, in the future, in the design process.

The solution proposed in this study, to reduce the pressure oscillation amplitude, and thereby increase the turbine operating range and flexibility, is to control the flow in an active manner, with kinematic energy supply, obtained through the controlled pitching movement of the guide vanes. This procedure can reduce the pressure oscillation amplitude at critical operating conditions, allowing the machine to operate in part load more smoothly and increasing the operating range, with the advantage of low or even beneficial impact on the turbine efficiency, as discussed by Wunderer AND Schilling [2]. The reduction of the pressure oscillation amplitude can also reduce the plant maintenance costs and outage periods, thanks to the consequently extended fatigue life of the mechanical components.

Besides the benefits of grid regulation offered by the extension of the operating range of hydraulic turbines, the possibility to operate at deep part load can, in some cases, specially in plants with a reduced number of generator units, lead to an additional annual energy output from approximately $5 \%$ up to $10 \%$.

\section{Numerical Simulations}

To develop and investigate the technique of active flow control, through the pitching movement of the turbine guide vanes, a complete Francis turbine, with $n_{q} \cong 80 \mathrm{~min}^{-1}$, was numerically simulated with CFD (computational fluid dynamics). This particular machine, denominated FT 80, was chosen, since Francis machines with high specific speeds are especially affected by the adverse operating conditions at part load. The numerical simulation offered an economic possibility to test the new control technique at this early development stage, prior to actual model

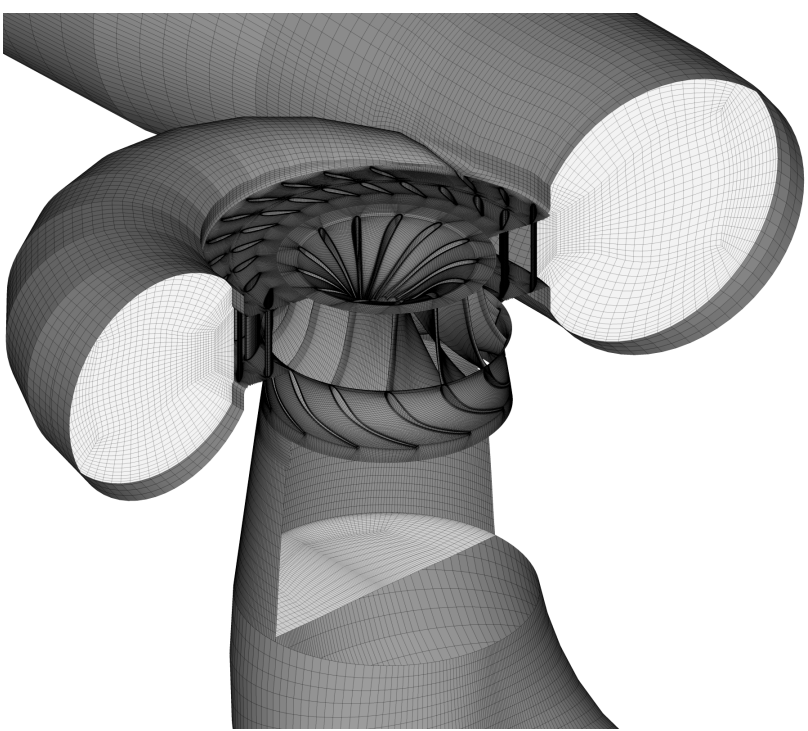

Figure 1: Computational mesh for the fluid flow simulation.

testing, with the additional advantage of offering practical visualisation and measuring tools, which might require complicated and expensive experimental devices.

The simulated operating points were taken from real operating conditions from an existing prototype turbine, already in operation and built with exact the same geometry as the turbine model FT 80 . The considered operating points comprise the optimum, full load, normal operation, part load at low and high heads and extreme part load. For the objective of this study, i.e. to extend the machine operating range, the last three load conditions are the most interesting and belong to the critical portion of the hill chart, in terms of pressure oscillations.

The computational model is only suitable for the investigations, if it can accurately reproduce the real behaviour of the physical flow through the turbine, qualitatively as well as quantitatively. Therefore, after the preparation of the numerical model, it was preliminary verified with stationary simulations, whose results were compared to the experimental head, flow, power and efficiency measurements available from the model test. As long as the main interest of the simulations was in reproducing the transient phenomena, the instationary calculations were also verified with the observations and pressure oscillation values from the experimental data.

\section{A. Stationary Simulations}

The numerical simulations were carried out with the finite volume method (FVM), applied to the complete turbine, with all its hydraulic components: spiral case, stay vanes, guide vanes, runner and draft tube. The calculations were conducted in a coupled manner, i.e. considering all the turbine components and interfaces 
Table I: Experimental results obtained at the model test and numerically simulated results.

\begin{tabular}{|c|c|c|c|c|c|c|c|c|c|c|c|c|}
\hline \multirow[b]{2}{*}{ Operating Point } & \multicolumn{4}{|c|}{ Model Test } & \multicolumn{4}{|c|}{ Simulation } & \multicolumn{4}{|c|}{ Deviation } \\
\hline & 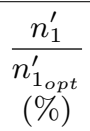 & $\begin{array}{c}\frac{Q_{1}^{\prime}}{Q_{1_{\text {opt }}^{\prime}}^{\prime}} \\
(\%)\end{array}$ & $\frac{T_{1}^{\prime}}{T_{1_{o p t}}^{\prime}}$ & $\begin{array}{c}\frac{\eta}{\eta_{o p t}} \\
(\%)\end{array}$ & 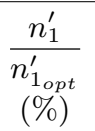 & $\begin{array}{c}Q_{1}^{\prime} \\
Q_{1_{o p t}^{\prime}}^{\prime} \\
(\%)\end{array}$ & $\frac{T_{1}^{\prime}}{T_{1_{o p t}}^{\prime}}$ & $\begin{array}{c}\frac{\eta}{\eta_{o p t}} \\
(\%)\end{array}$ & $\begin{array}{l}\delta n_{1}^{\prime} \\
(\%)\end{array}$ & $\begin{array}{l}\delta Q_{1}^{\prime} \\
(\%)\end{array}$ & $\begin{array}{l}\delta T_{1}^{\prime} \\
(\%)\end{array}$ & $\begin{array}{l}\delta \eta \\
(\%)\end{array}$ \\
\hline Optimum & 100,0 & 100,0 & 100,0 & 100,0 & 99,4 & 100,0 & 100,3 & 100,2 & $-0,6$ & $-0,6$ & 0,3 & 0,2 \\
\hline Rated & 110,3 & 122,9 & 108,3 & 97,2 & 109,6 & 122,2 & 110,0 & 98,7 & $-0,6$ & $-0,6$ & 1,6 & 1,5 \\
\hline Normal & 107,0 & 110,2 & 102,2 & 99,3 & 106,0 & 109,2 & 102,4 & 99,4 & $-1,0$ & $-1,0$ & 0,2 & 0,1 \\
\hline High Head & 107,8 & 79,7 & 69,3 & 93,8 & 106,7 & 78,9 & 69,3 & 93,8 & $-1,0$ & $-1,0$ & 0,0 & 0,0 \\
\hline Low Head & 119,9 & 84,8 & 64,4 & 91,0 & 119,0 & 84,2 & 65,1 & 91,9 & $-0,7$ & $-0,7$ & 1,0 & 1,0 \\
\hline
\end{tabular}

between them, as long as the dynamic effects in the fluid flow through the turbine arise from the interaction between its components, caused by the rotating runner and the stationary parts.

Different computational mesh densities were tested and optimized to deliver accurate results with acceptable computation times. The final computational mesh counted with more than 6 million cells and part of it can be seen in Figure 1. The employed FVM made use of second-order interpolation schemes.

With the preliminary stationary simulations, different turbulence models were tested: standard $k-\epsilon, k-\epsilon$ LCL, $k-\omega$ and $k-\omega$ SST, and no significant influence on the results was noticed. However, the standard $k-\epsilon$ model achieved the best agreement with the available experimental results. The comparison between the results from the computational simulation and from the model test is reproduced in Table I. The accuracy provided by the numerical model offers a maximum deviation under $2 \%$.

\section{B. Transient Simulations}

For the simulation of the instationary effects in the fluid flow through the turbine, like the rotor-stator interaction and the flow instabilities in the draft tube, adequate time resolution and turbulence models were needed. Approximately 400 time steps were calculated for each machine revolution, in order to capture not only the effects from the draft tube instabilities, but also from the rotor-stator interaction.

Considering the turbulence modelling, the URANS (unsteady averaged Navier-Stokes equations) model introduced excessive artificial dissipation in the flow simulation, as expected, and turned out to be unable to reproduce the highly transient effects in the turbine, especially in the draft tube. Therefore, adequate and more sophisticated turbulence models had to be employed. The detached eddy simulation (DES), for example, attempts to resolve the turbulent eddies, which are larger then the mesh resolution, as it would be done in a strict large eddy simulation (LES). On the other hand, the eddies smaller than the grid resolution are modelled, as done in URANS. This hybrid behaviour is achieved with the modification of the turbulence dissipation, $\epsilon$, with the limiter $F_{\mathrm{DES}}$, which accounts for the local mesh size. STRELETs [3], modified the $k-\omega$ SST model with the DES approach, coming to the modified turbulence transport equations below.

$$
\begin{aligned}
\frac{\partial(\rho k)}{\partial t}+\frac{\partial\left(\bar{u}_{j} \rho k\right)}{\partial x_{j}} & =\tau_{i j} \frac{\partial \bar{u}_{i}}{\partial x_{j}}-\beta^{*} \rho \omega k F_{D E S} \\
& +\frac{\partial}{\partial x_{j}}\left[\left(\mu+\sigma_{k} \mu_{T}\right) \frac{\partial k}{\partial x_{j}}\right] \\
\frac{\partial(\rho \omega)}{\partial t}+\frac{\partial\left(\bar{u}_{j} \rho \omega\right)}{\partial x_{j}} & =\frac{\gamma}{\nu_{T}} \tau_{i j} \frac{\partial \bar{u}_{i}}{\partial x_{j}}-\beta \rho \omega^{2} \\
& +\frac{\partial}{\partial x_{j}}\left[\left(\mu+\sigma_{\omega} \mu_{T}\right) \frac{\partial \omega}{\partial x_{j}}\right] \\
& +2\left(1-F_{1}\right) \rho \sigma_{\omega_{2}} \frac{1}{\omega} \frac{\partial k}{\partial x_{j}} \frac{\partial \omega}{\partial x_{j}}
\end{aligned}
$$

The influence of the mesh resolution appears in the dissipation term, $\beta^{*} \rho \omega k F_{D E S}$, in the turbulence kinetic energy transport equation. The last term in the transport equation of the specific dissipation rate is the normal SST modification of the original $k-\omega$ model. The interpolation scheme has also to be modified for the application of the DES model. In the regions where DES assumes the LES characteristic, the interpolation function must reproduce the CDS scheme. While, in other regions, where the URANS behaviour is dominant, the interpolation function must be second-order UDS. This blend between these two interpolations schemes is based on local flow characteristics and is described by TRAVIN [4].

The transient numerical simulation was also verified with experimental data. The calculated and measured pressure fluctuation amplitude at four measuring points at the draft tube cone were compared and are presented in Table II. The shape of the vortex rope in the draft tube could also be accurately predicted by the numerical simulation, as seen in Figure 2.

The transient simulation required approximately 30 machine revolutions, depending on the operating point and on the prescribed initial solution, until the instationary flow patterns were established. The computation of one machine revolution took, in average, 15 hours in a Linux cluster with 8 Intel Q6600 processors, each with 4 kernels, $2,4 \mathrm{GHz}$ and $2 \mathrm{~GB}$ memory. 
Table II: Experimental results obtained at the model test and numerically simulated with DES.

\begin{tabular}{|c|c|c|c|c|c|c|c|c|c|c|c|c|c|c|c|}
\hline \multirow[b]{3}{*}{ Operating Point } & \multicolumn{5}{|c|}{ Model Test } & \multicolumn{5}{|c|}{ Simulation } & \multicolumn{5}{|c|}{ Deviation } \\
\hline & \multicolumn{4}{|c|}{$\Delta p / \rho g H$} & \multirow{2}{*}{$\begin{array}{l}\frac{f}{f_{n}} \\
(-)\end{array}$} & \multicolumn{4}{|c|}{$\Delta p / \rho g H$} & \multirow{2}{*}{$\begin{array}{l}\frac{f}{f_{n}} \\
(-)\end{array}$} & \multicolumn{4}{|c|}{$\delta \Delta p$} & \multirow{2}{*}{$\begin{array}{l}\delta f \\
(\%)\end{array}$} \\
\hline & $\begin{array}{l}\text { HW } \\
(\%)\end{array}$ & $\begin{array}{l}90^{\circ} \\
(\%)\end{array}$ & $\begin{array}{l}\text { TW } \\
(\%)\end{array}$ & $\begin{array}{c}270^{\circ} \\
(\%)\end{array}$ & & $\begin{array}{l}\text { HW } \\
(\%)\end{array}$ & $\begin{array}{l}90^{\circ} \\
(\%)\end{array}$ & $\begin{array}{l}\text { TW } \\
(\%)\end{array}$ & $\begin{array}{c}270^{\circ} \\
(\%)\end{array}$ & & $\begin{array}{l}\text { HW } \\
(\%)\end{array}$ & $\begin{array}{l}90^{\circ} \\
(\%)\end{array}$ & $\begin{array}{l}\mathrm{TW} \\
(\%) \\
\end{array}$ & $\begin{array}{c}270^{\circ} \\
(\%)\end{array}$ & \\
\hline High Head & 4,70 & 3,65 & 4,28 & 5,25 & 0,302 & 4,31 & 3,23 & 3,85 & 4,52 & 0,298 & $-8,3$ & $-11,5$ & $-10,0$ & $-13,9$ & $-1,3$ \\
\hline Low Head & 5,29 & 4,51 & 5,71 & 6,78 & 0,282 & 5,04 & 4,44 & 5,94 & 6,40 & 0,315 & $-4,7$ & $-1,6$ & 4,0 & $-5,6$ & 11,7 \\
\hline
\end{tabular}
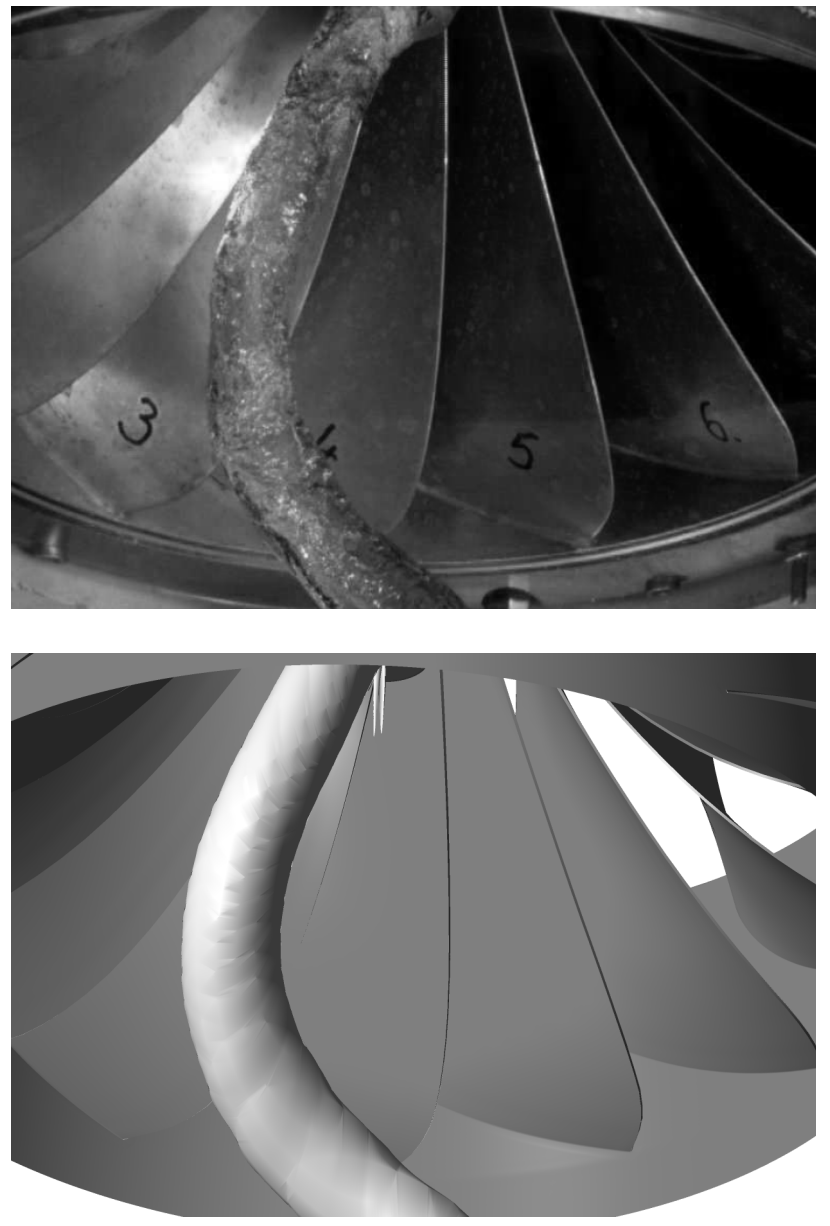

Figure 2: Comparison of the vortex rope shape observed at the model test and numerically simulated at part load and high head.

\section{Pitching Guide Vanes}

The active control of the flow through the turbine was reached with the pitching movement of the guide vanes. To the stationary opening angle of the guide vanes, $\Delta \gamma$, a harmonic oscillating component was added, $\delta(\Delta \gamma)$, in which the control parameters are the pitching angle amplitude, $\Delta \tilde{\gamma}$, and the pitching frequency, $\tilde{f}$.

$$
\delta(\Delta \gamma)=\Delta \tilde{\gamma} \sin \left[\tilde{f}\left(t-t_{0}\right)\right]
$$

At the begin of the simulation, the fluid volume in the distributor was meshed with the original stationary position of the guide vanes. With the pitching movement of the guide vanes, the mesh boundaries, which correspond to the guide vane walls, must be accordingly modified, to simulate the motion effect in the numerical computations. Still, the displacement of the mesh boundaries, associated to the guide vane walls, had to be followed by mesh adjustments in the surrounding region, in order to preserve the computational grid quality.

Here, the mesh deformation was specified, based on the displacement diffusion principle, which counts with a diffusion transport equation for the displacement in the computation field and assumes the prescribed movement of the guide vanes walls as the boundary conditions.

$$
\frac{\partial}{\partial x_{i}}\left(\Gamma_{\delta} \frac{\partial \delta}{\partial x_{i}}\right)=0
$$

This equation involves the local displacement diffusion parameter, $\Gamma_{\delta}$, which specifies how fast the displacement of the mesh boundaries are diffused into the computational grid. Its importance is limited to the numerical simulation and does not reflect any physical property of the real problem.

To limit the mesh deformation near to the wall boundaries and preserve the mesh resolution and quality in the boundary layer, the local diffusion is inversely proportional to the distance to the moving boundary, $\Gamma_{\delta}=(1 / d)^{K}, K>0$. It has the effect, that the mesh near to the moving wall remains almost undeformed, while the stream region absorbs the most part of the mesh deformation caused by the displacement of the mesh boundaries.

\section{Application}

\section{A. Pressure Oscillation Amplitude}

After the verification of the numerical model accuracy and its application to the simulation of several turbine operating points, with stationary guide vanes, it was used for the simulation of the effect of pitching guide vanes on the fluid flow.

Figure 3 shows the massive vortices in the runner blades channel on the suction side of the blades at extreme part load. This critical operating point results in strong pressure pulsations, which would prevent the runner from being operated at this condition. The objective with the introduction of the active flow 


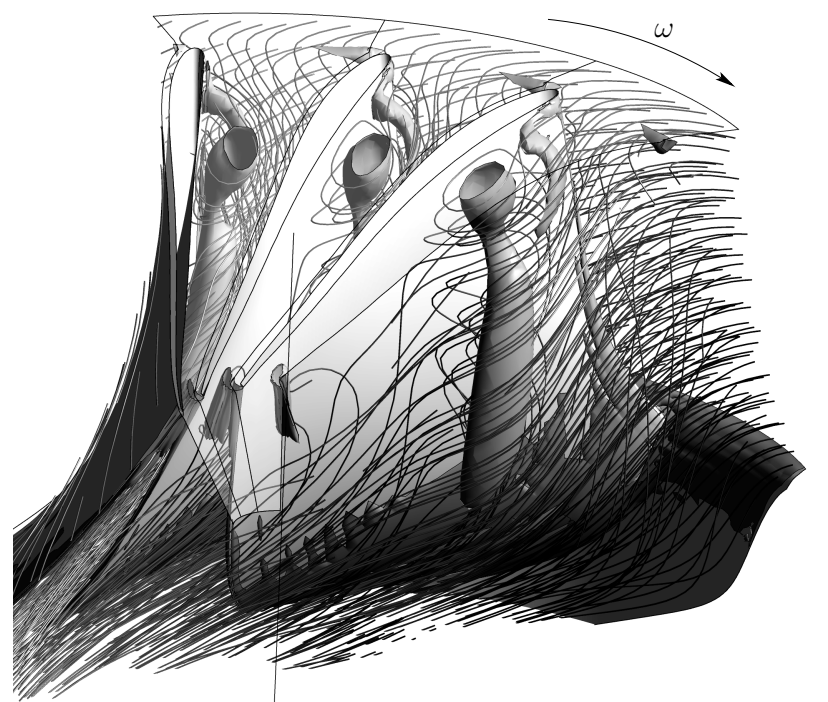

Figure 3: Streamlines and interchannel vortex at the turbine runner at deep part load and high head.

control, though the pitching guide vanes, is to reduce the pressure pulsations at operating conditions, such as the one just mentioned, in order to extent the turbine operating range, increasing the annual energy output.

WUNDERER [5] commented on the possibility to successfully employ this kind of active flow control to reduce the pressure pulsations in Francis turbines with high specific speed. However, his numerical experiments were limited to extremely simplified models and very singular operating points and blade geometry configurations. To test the actual effectiveness of this technique in real turbines, different control parameters $\Delta \tilde{\gamma}$ and $\tilde{f}$, for the pitching movement of the guide vanes, were tested with the computational fluid flow model of the FT 80 machine, whose high reliability for dynamic simulations was presented in the previous section.

The active flow control was simulated for the FT 80 turbine, at part load and low head, $H / H_{o p t}=0,696$ and $Q / Q_{o p t}=0,707$. First, the pitching amplitude was set to $\Delta \tilde{\gamma}=0,50^{\circ}$ and its frequency to $\tilde{f} / f_{n}=1,5$, where $f_{n}$ is the turbine rotation frequency. Afterwards, the pitching frequency, $\tilde{f}$, was reduced and synchronized to the vortex rope rotating frequency, $f / f_{n}=0,31$, and two different amplitudes were tested, $\Delta \tilde{\gamma}=0,50^{\circ}$ and $\Delta \tilde{\gamma}=0,75^{\circ}$.

The influence of the active flow control on the pressure pulsations in the turbine runner, with different pitching amplitudes and the pitching frequency synchronized to the vortex rope rotating frequency, $f / f_{n}=0,31$, can be observed in Figure 4. The pressure pulsation amplitude, normalized in relation to the head, $\Delta p / \rho g H$, is shown at three conformal planes: near to the crown, $v=0,025$, at the distributor centre line, $v=0,500$, and near to the band, $v=0,975$. The pressure pulsation amplitude is plotted along the developed blade length, $u$, at these conformal planes, beginning at the trailing edge at the pressure side
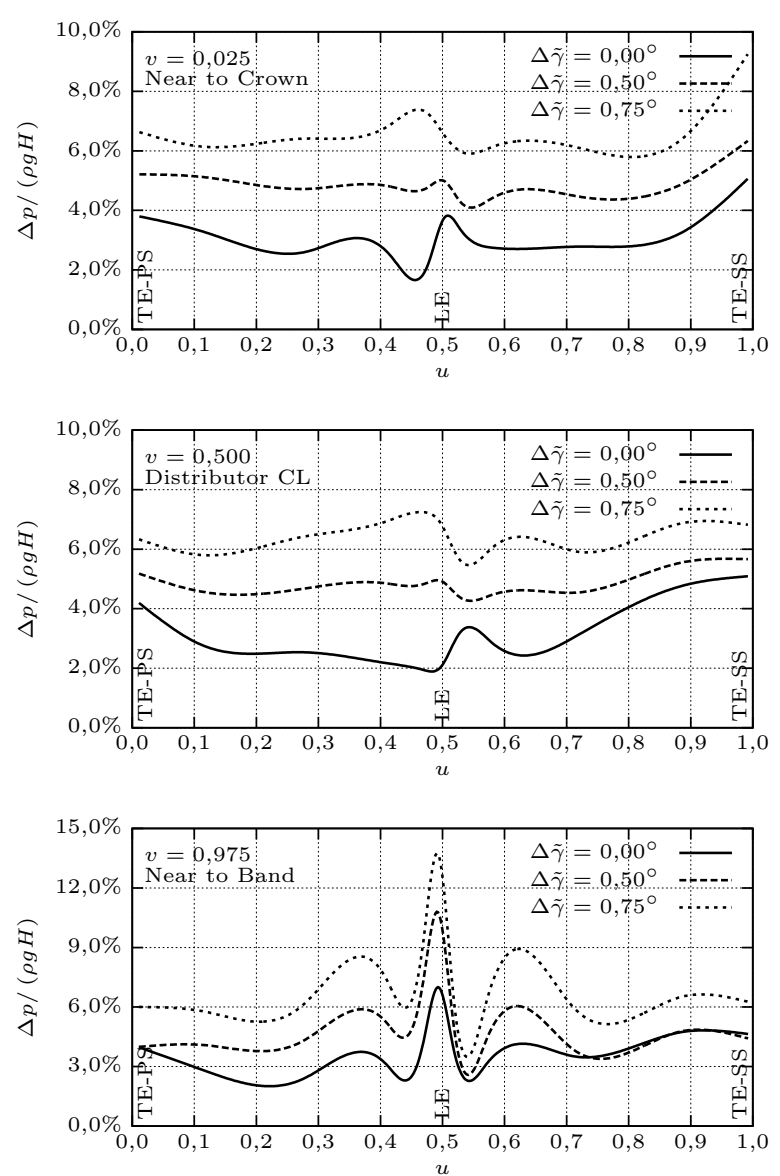

Figure 4: Pressure pulsation amplitude along the blade developed length, $u$, at different conformal planes, $v=0,025, v=0,500$ and $v=0,975$, as function of the guide vanes pitching amplitude, $\Delta \tilde{\gamma}=0,50^{\circ}$ and $\Delta \tilde{\gamma}=0,75^{\circ}$, at constant pitching frequency, $\tilde{f} / f_{n}=0,31$.

(TE-PS), $u=0,0$, passing by the blade leading edge (LE), $u=0,5$, and finishing at the trailing edge at the suction side (TE-SS), $u=1,0$. At each plot, there are curves for the numerical simulations with stationary guide vanes, $\Delta \tilde{\gamma}=0$, and pitching guide vane amplitudes of $\Delta \tilde{\gamma}=0,50^{\circ}$ and $\Delta \tilde{\gamma}=0,75^{\circ}$.

As it can be seen in Figure 4, the numerical simulations demonstrate that the guide vanes pitching movement, in the same frequency as the draft tube vortex, rather intensifies the pressure pulsations in the runner, producing exactly the opposite effect as desired. It also suggest, based on the curves for $\Delta \tilde{\gamma}=0,50^{\circ}$ and $\Delta \tilde{\gamma}=0,75^{\circ}$, that the increase of the pitching amplitude at this frequency also intensifies the severity of the pressure pulsations.

On the other hand, Figure 5 exemplifies the effect of varying the guide vanes pitching frequency, while the pitching amplitude remains constant. In this plot, the pressure pulsation is normalized in relation to the values obtained with stationary guide vanes. In the numerical experiments, the frequency assumed the values $\tilde{f} / f_{n}=0, \tilde{f} / f_{n}=0,31$ and $\tilde{f} / f_{n}=1,5$, and the amplitude was $\Delta \tilde{\gamma}=0,50^{\circ}$. The curves presented in Figure 5 correspond to two measuring points at a 


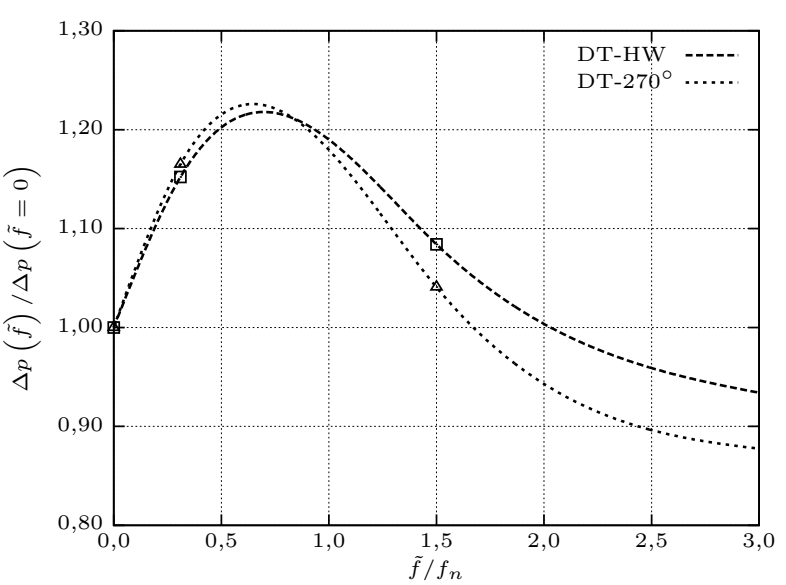

Figure 5: Pressure pulsation amplitude at draft tube measuring points, DT-TW and DT- $270^{\circ}$, as function of the guide vanes pitching frequency, $\tilde{f} / f_{n}=0,31$, at constant pitching amplitude, $\Delta \tilde{\gamma}=0,50^{\circ}$.

circular section of the draft tube cone, at the tail water side (DT-TW) and at a quarter circle offset (DT-270).

Supposing that the dynamic characteristic of the excited system is similar to a second-order damped dynamic system, the pressure pulsation curves as functions of the pitching frequency were extrapolated up to $\tilde{f} / f_{n}=3,0$. If in the future, with help of further numerical simulations, this assumption shows up to be correct, the increase of the guide vanes pitching frequency could possibly reduce the pressure oscillation amplitude, resulting in the desired effect in order to extent the machine operating range. Nevertheless, this dynamic behaviour as a function of the pitching frequency is by now just a hypothesis and has to be confirmed by future numerical experiments, with several combinations of the control parameters, $\Delta \tilde{\gamma}$, $\tilde{f} / f_{n}$, and at different operating points. Supposing that this assumption is correct, it shall also be verified that the guide vanes pitching frequency is practicable, from the point of view of the mechanical design.

\section{B. Energy Output}

Here it is assumed that the introduction of the active flow control, through pitching guide vanes with optimal control parameters, will be able to reduce the pressure pulsations up to a point that moderate part load conditions can be added to the turbine operation range. As planned before, this extension of the machine operating flexibility increases the possibility to offer auxiliary services to the electrical grid and the annual energy output.

Taking as example an existing hydraulic powerplant with four 150-MW Francis machines, with slightly higher specific speed as simulated, $n_{q} \cong 90 \mathrm{~min}^{-1}$, where the operation at part load would be desirable, but avoided because of strong pressure oscillations, the increase of the annual energy output, with the application of the active flow control, could reach $4,9 \%$,
Table III: Example of theoretical gains applied to an existing powerplant.

\begin{tabular}{lr}
\hline Machine & FT 90 \\
\hline Number of Machines & $z=4$ \\
Rated Power per Machine & $P_{r}=150 \mathrm{MW}$ \\
Original Lower Power Limit & $P_{\min }=0,70 P_{r}$ \\
\hline Extended Lower Power Limit & $P_{\min }=0,45 P_{r}$ \\
Increased Availability & $+7,5 \%$ \\
Increased Annual Energy Output & $+4,8 \%$ \\
\hline
\end{tabular}

through an increased availability of 7,5\%. The machine data for this real example and the operational gains can be found in Table III.

\section{Conclusion}

The first numerical simulations with pitching guide vanes, in the particular case of the FT 80 turbine, pointed out that the possibility of reducing the pressure pulsations in the machine, at part load, requires further numerical simulation and experiments. The reduction of the pressure oscillation amplitude would allow to operate the turbine in severe load conditions, without compromising its mechanical structure and extending its operating range. The additional operational flexibility, which would possibly be achieved with the proposed technique, would increase the offer for regulation services and would expand the annual energy output, in some hydraulic powerplants, around $5 \%$ up to $10 \%$. Other renewable energy sources, such as wind, solar, wave and tidal energy, could also profit from the extended capability of water turbines to regulate the electrical grid. Further investigations in the development of the active flow control, with pitching guide vanes, shall be the simulation of additional operating points, of machines with different specific speeds and of various sets of control parameters.

\section{References}

[1] M. V. Magnoli and R. Schilling. Vortex Shedding in Francis Runners Trailing Edges. In Proceedings of the 24th IAHR Symposium, Foz do Iguaçu, Brazil, 2008.

[2] R. Wunderer and R. Schilling. Numerical simulation of active flow control in hydro turbines. In 12th ISROMAC, Honolulu, Hawaii, USA, 2000.

[3] M. Strelets. Detached Eddy Simulation of Massively Separated Flows. In AIAA, editor, 39th Aerospace Sciences Meeting and Exhibit, volume Paper 2001-0879, Reno, Nevada, USA, 2001.

[4] A. Travin, M. Shur, M. Strelets, and P. R. Spalart. Physical and numerical upgrades in the detached-eddy simulation of complex turbulent flows. In 412 EUROMECH Colloquium, München, Germany, 2000.

[5] R. Wunderer. Numerische Simulation der aktiven Stömungsbeeinflussung in Turbomaschinen. $\mathrm{PhD}$ thesis, Technische Universität München, Lehrstuhl für Fluidmechanik, 2010. 